\title{
CME: Dermatology (135279): self-assessment questionnaire
}

\author{
Authors: Edited by Emma Benton and Tahseen A Chowdhury
}

\section{DOI: $10.7861 /$ clinmed.SAQ.21.3}

\section{SAQs and answers are ONLINE for RCP fellows and collegiate members}

Format

Candidates are asked to choose the best answer from the five possible answers. This best of five format is used in many medical examinations; however, the questions are not intended to be representative of those used in the MRCP(UK) Part 1 or Part 2 Written Examinations.

\section{The answering process}

1 Go to https://cme.rcplondon.ac.uk

2 Log on using your usual RCP username and password

3 Select the relevant CME question paper

4 Answer all 10 questions by selecting the best answer from the options provided

5 Once you have answered all the questions, click on Submit

\section{Registering your external CPD credits}

Carrying out this activity allows you to claim two external CPD credits. These will be automatically transferred to your CPD diary, where you can review the activity and claim your points.

1. A 63-year-old man presented with a 3-month history of recurrent mouth ulcers and skin lesions on the trunk and limbs. Examination demonstrated multiple shallow erosions on the trunk and irregular ulcers on the buccal mucosa. Direct immunofluorescence performed on a perilesional skin biopsy section demonstrated intraepithelial immunoglobulin (Ig) G binding with acantholysis. A diagnosis of pemphigus vulgaris was made.

Which protein is the target of pathogenic antibodies in this condition?
(a) Desmoglein 1.
(b) Desmoglein 3.
(c) Periplakin.
(d) Envoplakin.
(e) Type VII collagen.

2. A 78-year-old woman presented with a 6-month history of blisters and itchy skin lesions. She had a background of type 2 diabetes and was taking linagliptin. On examination, a number of urticated plaques were noted on the trunk and limbs with tense bullae and erosions. Examination of the mouth and other mucosal surfaces was unremarkable. Immunofluorescence performed on a perilesional skin biopsy demonstrated IgG binding to the basement membrane of the epidermis.

\section{What is the most likely diagnosis?}

(a) Bullous pemphigoid.

(b) Dermatitis herpetiformis.

(c) Epidermolysis bullosa aquisita.

(d) Pemphigus foliaceus.

(e) Pemphigus vulgaris.

3. A 43-year-old woman presented with intensely itchy lesions on the arms and buttocks. She also gave a history of change in bowel habit over the past year. On examination there were numerous small crusted erosions on the extensor forearms and buttocks. Examination of the mouth and other mucosal surfaces was unremarkable. Direct immunofluorescence demonstrated granular IgA deposition in the upper dermis. A diagnosis of dermatitis herpetiformis was made.

\section{What other disease is this most associated with?}
(a) Coeliac disease.
(b) Crohn's disease.
(c) Hirschsprung disease.
(d) Pernicious anaemia.
(e) Ulcerative colitis.

4. Wilson's disease can lead to a build-up of copper within the nail plate, and a change in colour of the lunula.

\section{What colour lunulae could indicate this disease?}
(a) Blue.
(b) Gold.
(c) Green.
(d) Orange.
(e) Pink. 
5. Which of these cutaneous manifestations of SARS-CoV-2 would indicate more severe COVID-19 disease?
(a) COVID toes.
(b) Blistering chicken pox-like rash.
(c) Livedoid rash.
(d) Maculopapular rash.
(e) Urticarial rash.

6. Scabies can be transmitted in which of the following circumstances?
(a) Fomites (such as bedding) for up to 1 week.
(b) Frequently from dogs as sarcoptic mange.
(c) Mites can jump from person to person.
(d) Skin-to-skin contact for 1 minute.
(e) Skin-to-skin contact for 20 minutes.

\section{Which of the following is a screening tool for psoriatic} arthritis?
(a) Dermatology Life Quality Index (DLQI).
(b) Hospital Anxiety and Depression Scale (HADS).
(c) Psoriasis Area and Severity Index (PASI).
(d) Psoriasis Epidemiology Screening Tool (PEST).
(e) PSORS1

8. A 36-year-old woman with chronic plaque psoriasis presented with worsening skin disease despite optimised topical therapy. She was started on methotrexate.

\section{Which of the following is true regarding methotrexate therapy?}

(a) Methotrexate is taken once a day.

(b) Patients may receive yellow fever vaccination while on methotrexate.

(c) Subcutaneous methotrexate causes more gastrointestinal side effects than oral methotrexate.

(d) Bone marrow suppression is a possible complication.

(e) Methotrexate should be continued during pregnancy.

9. A 48-year-old man presented with fever and a painful rash. He was known to have a history of eczema, since childhood, and had been visiting his parents. Examination revealed generalised erythema and scale, and a vesicular rash across one side of the face. Bloods showed a white cell count of $11.2 \times 10^{9} / \mathrm{L}$, eosinophil count of $0.9 \times 10^{9} / \mathrm{L}$ and C-reactive protein of $112 \mathrm{mg} / \mathrm{L}$.
What is the most likely cause of his atopic dermatitis flare?
(a) Contact allergy to his parents' new dog.
(b) Drug reaction with eosinophilia and systemic symptoms (DRESS).
(c) Herpes simplex infection.
(d) Molluscum contagiosum.
(e) Viral upper respiratory tract infection.

10. A 56-year-old woman with lifelong, moderate-to-severe atopic dermatitis was seen in the dermatology clinic to discuss treatment. She had a history of non-alcoholic fatty liver disease and type 2 diabetes mellitus. She had previously failed methotrexate due to deranged liver function tests while on treatment. She was switched to dupilumab 6 months ago but had been struggling with debilitating bilateral conjunctivitis, a recognised effect of dupilumab treatment. The possibility of abrocitinib was discussed through the early access to medicine scheme.

\section{What is the mechanism of action of abrocitinib?}
(a) IL4/13 receptor blockade.
(b) Immunoglibulin E inhibitor.
(c) Inhibits folate reductase.
(d) JAK 1 and 2 inhibitor.
(e) JAK 1 inhibitor.

\section{CME Gastroenterology SAQ} Answers to the CME SAQ published in Clinical Medicine in March 2021

$\begin{array}{llllllllll}\text { Q1 } & \text { Q2 } & \text { Q3 } & \text { Q4 } & \text { Q5 } & \text { Q6 } & \text { Q7 } & \text { Q8 } & \text { Q9 } & \text { Q10 } \\ \text { (e) } & \text { (c) } & \text { (c) } & \text { (e) } & \text { (e) } & \text { (c) } & \text { (a) } & \text { (c) } & \text { (e) } & \text { (e) }\end{array}$

